\title{
INFORMAÇÕES EDITORIAIS 2011
}

\begin{tabular}{|c|c|c|c|c|c|c|}
\hline \multicolumn{7}{|c|}{ ARTIGOS PUBLICADOS } \\
\hline \multirow[t]{2}{*}{ Edições } & \multicolumn{4}{|c|}{ Número de Artigos } & \multirow{2}{*}{$\begin{array}{l}\text { Artigos por } \\
\text { Edição }\end{array}$} & \multirow{2}{*}{$\begin{array}{c}\text { Artigos } \\
\text { Reprovados }\end{array}$} \\
\hline & UFPR & $\%$ & OUTRAS & $\%$ & & \\
\hline $\begin{array}{l}\text { Jan/Abr, } \\
2011\end{array}$ & 1 & $17 \%$ & 5 & $83 \%$ & 6 & 3 \\
\hline $\begin{array}{l}\text { Mai/Ago, } \\
2011\end{array}$ & 1 & $17 \%$ & 5 & $83 \%$ & 6 & 5 \\
\hline $\begin{array}{l}\text { Set/Dez, } \\
2011\end{array}$ & 0 & 0 & 6 & 100 & 6 & 7 \\
\hline Média & 0,67 & $11,33 \%$ & 5,33 & $88 \%$ & 6 & 15 \\
\hline
\end{tabular}

\begin{tabular}{l|c|c|c|c}
\hline \multirow{2}{*}{ Edições } & \multicolumn{3}{|c|}{ Número de Autores } & \multirow{2}{*}{ Total de Autores } \\
\cline { 2 - 4 } & UFPR & PR & OUTRAS UF's & \\
\hline Jan/Abr, 2010 & & & 11 & 11 \\
\hline Mai/Ago, 2010 & 3 & 3 & 14 & 17 \\
\hline Set/Dez, 2010 & 1 & 8 & 12 & 20 \\
\hline Média/total & $\mathbf{1 , 3 3 3 3}$ & $\mathbf{3 , 6 7}$ & $\mathbf{1 2 , 3 3}$ & $\mathbf{4 8}$ \\
\hline
\end{tabular}

\section{DEMANDA POR PUBLICAÇÃO}

\begin{tabular}{l|c|c}
\multicolumn{1}{c|}{ Artigos } & \multicolumn{2}{c}{$\begin{array}{c}\text { Período Janeiro a } \\
\text { Dezembro/2011 }\end{array}$} \\
\hline Recebidos & 51 & 100 \\
\hline Aprovados & 18 & $35,29 \%$ \\
\hline Não Aprovados (após Blind review) & 12 & $23,53 \%$ \\
\hline Não aceitos para avaliação & 5 & $9,80 \%$ \\
\hline Em avaliação & 16 & $31,37 \%$ \\
\hline
\end{tabular}


R C \& C

REVISTA DE CONTABILIDADE E CONTROLADORIA

\begin{tabular}{|c|c|c|c|c|c|c|}
\hline \multicolumn{7}{|c|}{ NUMERO DE AUTORES POR UNIDADE DE FEDERAÇÃO } \\
\hline \multirow[t]{3}{*}{ Edições } & \multicolumn{2}{|c|}{ Jan/Abr, 2011} & \multicolumn{2}{|c|}{ Mai/Ago, 2011} & \multicolumn{2}{|c|}{ Set/Dez, 2011} \\
\hline & $\mathrm{Fi}$ & $\mathrm{Fi} \%$ & $\mathrm{Fi}$ & $\mathrm{Fi} \%$ & $\mathrm{Fi}$ & $\mathrm{Fi} \%$ \\
\hline & 5 & $33,33 \%$ & 4 & $20,00 \%$ & 1 & $8 \%$ \\
\hline Santa Catarina & 3 & $20,00 \%$ & 7 & $35,00 \%$ & & \\
\hline Rio Grande do Sul & & & & & 4 & $33 \%$ \\
\hline Minas Gerais & 3 & $20,00 \%$ & 4 & $20,00 \%$ & 2 & $17 \%$ \\
\hline Ceará & & & & & 2 & $17 \%$ \\
\hline Rio de Janeiro & 1 & $6,67 \%$ & 3 & $15,00 \%$ & & \\
\hline São Paulo & 1 & $6,67 \%$ & & & 3 & $25 \%$ \\
\hline Bahia & 2 & $13,33 \%$ & & & & \\
\hline Espírito Santo & & & 2 & $10,00 \%$ & & \\
\hline Total & 15 & $100,00 \%$ & 20 & $100 \%$ & 12 & $100 \%$ \\
\hline
\end{tabular}

\begin{tabular}{|c|c|c|c|c|}
\hline \multicolumn{5}{|c|}{ INSTITUIÇÕES DE ORIGEM DOS ARTIGOS } \\
\hline \multirow[t]{2}{*}{ UF de origem } & \multicolumn{4}{|c|}{ Edições } \\
\hline & Jan/Abr, 2011 & Mai/Ago, 2011 & Set/Dez, 2011 & TOTAL \\
\hline UFPR-PR & 1 & 1 & & 2 \\
\hline UPS-SP & 1 & & & 1 \\
\hline FURB-SC & 1 & & & 1 \\
\hline UFMG-MG & 1 & 1 & & 2 \\
\hline UNITAU & & & 1 & 1 \\
\hline PUCMINAS-MG & & & 1 & 1 \\
\hline UNIOESTE-PR & & & 1 & 1 \\
\hline UFSC-SC & & 2 & & 2 \\
\hline UCS-RS & & & 1 & 1 \\
\hline UERJ-RJ & & & & 0 \\
\hline UFC-CE & & & 1 & 1 \\
\hline UFBA & 1 & & & 1 \\
\hline UFRJ & 1 & 1 & & 2 \\
\hline UFES-ES & & 1 & & 1 \\
\hline UNB-DF & & & 1 & 1 \\
\hline TOTAL & 6 & 6 & 6 & 18 \\
\hline
\end{tabular}




\section{PERIODO MÉDIO DE CIRCULAÇÃO DOS ORIGINAIS PUBLICADOS EM 2011}

\section{Vol. 3, N 1, 2011}

\begin{tabular}{|c|c|c|c|c|c|c|c|c|}
\hline $\begin{array}{l}\text { N. do } \\
\text { Artigo }\end{array}$ & $\begin{array}{c}\text { Data de } \\
\text { submissão }\end{array}$ & $\begin{array}{c}\text { Data da } \\
\text { Aceitação }\end{array}$ & $\begin{array}{l}\text { Reformulado } \\
\text { pelos autores }\end{array}$ & $\begin{array}{c}\text { Data da } \\
\text { Publicação }\end{array}$ & $\begin{array}{c}\text { N. de dias } \\
\text { para } \\
\text { avaliação }\end{array}$ & $\begin{array}{c}\text { N. de dias } \\
\text { para } \\
\text { reformulação }\end{array}$ & $\begin{array}{l}\text { N. de dias para } \\
\text { recomendação } \\
\text { de publicação }\end{array}$ & $\begin{array}{c}\text { N. de diias } \\
\text { entre a } \\
\text { submissão e } \\
\text { publicação }\end{array}$ \\
\hline 1 & 19.10.2010 & 13.11 .2010 & 20.12.2010 & 30.04 .2011 & 24 & 37 & 61 & 208 \\
\hline 2 & 23.10.2010 & 28.01.2011 & 28.04.2011 & 30.04 .2011 & 97 & 91 & 188 & 190 \\
\hline 3 & 25.09.2010 & 13.11.2010 & 20.12.2010 & 30.04 .2011 & 49 & 37 & 86 & 217 \\
\hline 4 & 19.11.2010 & 15.01.2011 & 03.02 .2011 & 30.04 .2011 & 57 & 19 & 76 & 163 \\
\hline 5 & 25.02.2010 & 13.07.2010 & 22.10.2010 & 30.04 .2011 & 140 & 101 & 241 & 429 \\
\hline 6 & 27.10 .2010 & 14.02 .2011 & 25.04 .2011 & 30.04 .2011 & 110 & 70 & 180 & 185 \\
\hline
\end{tabular}

\section{Vol. 3, N 2, 2011}

\begin{tabular}{|c|c|c|c|c|c|c|c|c|}
\hline $\begin{array}{l}\text { N. do } \\
\text { Artigo }\end{array}$ & $\begin{array}{c}\text { Data de } \\
\text { submissão }\end{array}$ & $\begin{array}{c}\text { Data da } \\
\text { Aceitação }\end{array}$ & $\begin{array}{l}\text { Reformulado } \\
\text { pelos autores }\end{array}$ & $\begin{array}{c}\text { Data da } \\
\text { Publicação }\end{array}$ & $\begin{array}{c}\text { N. de dias } \\
\text { para } \\
\text { avaliação }\end{array}$ & $\begin{array}{l}\text { N. de dias } \\
\text { para } \\
\text { reformulação }\end{array}$ & $\begin{array}{l}\text { N. de dias para } \\
\text { recomendação } \\
\text { de publicação }\end{array}$ & $\begin{array}{c}\text { N. de diias } \\
\text { entre a } \\
\text { submissão e } \\
\text { publicação }\end{array}$ \\
\hline 1 & 01.02.2011 & 04.04.2011 & 08.08 .2011 & 02.09.2011 & 63 & 127 & 190 & 214 \\
\hline 2 & 20.06.2010 & 20.07.2011 & 10.08.2011 & 02.09.2011 & 30 & 21 & 51 & 73 \\
\hline 3 & 28.02.2011 & 26.04.2011 & 17.06.2011 & 02.09.2011 & 57 & 52 & 109 & 187 \\
\hline 4 & 20.04.2011 & 13.06.2011 & 08.08.2011 & 02.09 .2011 & 57 & 56 & 113 & 138 \\
\hline 5 & 02.02 .2011 & 07.06 .2011 & 26.08.2011 & 02.09.2011 & 125 & 80 & 205 & 213 \\
\hline 6 & 01.02.2011 & 04.04 .2011 & 08.08.2011 & 02.09.2011 & 63 & 126 & 189 & 214 \\
\hline
\end{tabular}

\section{Vol. 3, N 3, 2011}

\begin{tabular}{|c|c|c|c|c|c|c|c|c|}
\hline $\begin{array}{l}\text { N. do } \\
\text { Artigo }\end{array}$ & $\begin{array}{c}\text { Data de } \\
\text { submissão }\end{array}$ & $\begin{array}{c}\text { Data da } \\
\text { Aceitação }\end{array}$ & $\begin{array}{l}\text { Reformulado } \\
\text { pelos autores }\end{array}$ & $\begin{array}{c}\text { Data da } \\
\text { Publicação }\end{array}$ & $\begin{array}{l}\text { N. de dias } \\
\text { para } \\
\text { avaliação }\end{array}$ & $\begin{array}{l}\text { N. de dias } \\
\text { para } \\
\text { reformulação }\end{array}$ & $\begin{array}{l}\text { N. de dias para } \\
\text { recomendação } \\
\text { de publicação }\end{array}$ & $\begin{array}{c}\text { N. de diias } \\
\text { entre a } \\
\text { submissão e } \\
\text { publicação }\end{array}$ \\
\hline 1 & 27.06.2011 & 04.08 .2011 & 02.09.2011 & 21.10.2011 & 38 & 25 & 63 & 113 \\
\hline 2 & 18.07.2011 & 17.09.2011 & 06.10 .2011 & 21.10.2011 & 62 & 24 & 86 & 102 \\
\hline 3 & 26.08.2011 & 29.09.2011 & 05.10 .2011 & 21.10.2011 & 95 & 7 & 102 & 103 \\
\hline 4 & 07.11 .2010 & 05.04 .2011 & 09.08.2011 & 21.10.2011 & 150 & 126 & 276 & 320 \\
\hline 5 & 01.02.2011 & 04.04 .2011 & 08.08.2011 & 21.10.2011 & 63 & 127 & 190 & 233 \\
\hline 6 & 20.06.2011 & 30.08 .2011 & 08.09 .2011 & 21.10.2011 & 72 & 10 & 82 & 126 \\
\hline
\end{tabular}




\begin{tabular}{c}
\hline AVALIADORES BRASILEIROS AD HOC E INSTITUIÇÃO DE \\
VÍNCULO QUE ATUARAM EM 2011 \\
\hline
\end{tabular}

\begin{tabular}{|c|c|}
\hline Avaliadores Brasileiros & Instituição \\
\hline Ana Paula Mussi Szabo Cherobim & Universidade Federal do Paraná \\
\hline Aneide de Oliveira Araujo & Universidade Federal do Rio Grande do Norte \\
\hline Antônio Artur de Souza & Universidade Federal de Minas Gerais \\
\hline Antonio Carlos Dias Coelho & Universidade Federal do Ceará \\
\hline Antonio Henriques de Araújo & Instituto Tecnológica da Aeronáutica \\
\hline Antonio Lopo Martinez & Fucape Business School \\
\hline Aracéli Cristina de Sousa Ferreira & Universidade Federal do Rio de Janeiro \\
\hline Carlos Renato Theóphilo & Universidade Estadual de Montes Claros \\
\hline Cláudio Parisi & Fundação Escola de Comércio Álvares Penteado \\
\hline Edgard Bruno Cornachione Junior & Universidade de São Paulo \\
\hline Eliseu Martins & Universidade de São Paulo \\
\hline Ernani Ott & Universidade do Vale do Rio dos Sinos \\
\hline Fábio Frezatti & Universidade de São Paulo \\
\hline Gilmar Almeida Gomes & Universidade do Estado de Santa Catarina \\
\hline Ilse Maria Beuren & Universidade Regional de Blumenau \\
\hline Jacqueline Veneroso Alves da Cunha & Universidade Federal de Minas Gerais \\
\hline José Alonso Borba & Universidade Federal de Santa Catarina \\
\hline Jose Luis Gomes da Silva & Universidade de Taubaté \\
\hline José Maria Dias Filho & Universidade Federal da Bahia \\
\hline Lauro Brito de Almeida & Universidade Federal do Paraná \\
\hline Léo Tadeu Robles & Pontificia Universidade Católica de Santos \\
\hline Luiz Carlos Miranda & Universidade Federal de Pernambuco \\
\hline Maísa de Souza Ribeiro & Universidade de São Paulo \\
\hline Márcia Martins Mendes De Luca & Universidade Federal do Ceará \\
\hline Marcos Antonio de Souza & Universidade do Vale do Rio dos Sinos \\
\hline Mariomar de Sales Lima & Universidade Federal do Amazonas \\
\hline Masayuki Nakagawa & Universidade de São Paulo \\
\hline Moacir Sancovschi & Universidade Federal do Rio de Janeiro \\
\hline Monica Franchi Carniello & Universidade de Taubaté \\
\hline Olga Maria Panhoca da Silva & Universidade do Estado de Santa Catarina \\
\hline Paulo Arnaldo Olak & Universidade Estadual de Londrina \\
\hline Paulo Roberto Barbosa Lustosa & Universidade Federal de Brasília \\
\hline
\end{tabular}




\begin{tabular}{l}
\hline AVALIADORES ESTRANGEIROS AD HOC QUE ATUARAM EM \\
2011 \\
\hline
\end{tabular}

\begin{tabular}{|l|l|l|l|}
\hline Avaliadores estrangeiros & Instituição & Cidade & País \\
\hline Carlos Alberto de Mello-e-Souza & Seattle University & Washington & Estados Unidos \\
\hline Francisco Carreira & Instituto Politécnico de Setúbal & Setúbal & Portugal \\
\hline
\end{tabular}

INSTITUIÇÕES DE LOTAÇÃO DOS AVALIADORES BRASILEIROS
QUE ATUARAM DEM 2011

\begin{tabular}{|l|c|c|c|}
\hline \multicolumn{1}{|c|}{ Instituição } & Estado & Quantidade & Percentual \\
\hline Fucape Business School & ES & 1 & 3,03 \\
\hline Fundação Escola de Comércio Álvares Penteado & SP & 1 & 3,03 \\
\hline Instituto Tecnológica da Aeronáutica & SP & 1 & 3,03 \\
\hline Pontificia Universidade Católica de Santos & SP & 1 & 3,03 \\
\hline Universidade de São Paulo & SP & 6 & 18,18 \\
\hline Universidade de Taubaté & SP & 2 & 6,06 \\
\hline Universidade do Estado de Santa Catarina & SC & 2 & 6,06 \\
\hline Universidade do Vale do Rio dos Sinos & RS & 2 & 6,06 \\
\hline Universidade Estadual de Londrina & PR & 1 & 3,03 \\
\hline Universidade Estadual de Montes Claros & MG & 1 & 3,03 \\
\hline Universidade Federal da Bahia & BA & 1 & 3,03 \\
\hline Universidade Federal de Brasília & DF & 1 & 3,03 \\
\hline Universidade Federal de Minas Gerais & MG & 2 & 6,06 \\
\hline Universidade Federal de Pernambuco & PE & 1 & 3,03 \\
\hline Universidade Federal de Santa Catarina & SC & 1 & 3,03 \\
\hline Universidade Federal do Amazonas & AM & 1 & 3,03 \\
\hline Universidade Federal do Ceará & CE & 2 & 6,06 \\
\hline Universidade Federal do Paraná & PR & 2 & 6,06 \\
\hline Universidade Federal do Rio de Janeiro & RJ & 2 & 6,06 \\
\hline Universidade Federal do Rio Grande do Norte & RN & 1 & 3,03 \\
\hline Universidade Regional de Blumenau & SC & 1 & 3,03 \\
\hline
\end{tabular}


LOCAL DE ORIGEM DOS AVALIADORES DE 2011

\begin{tabular}{|c|c|c|}
\hline Estado & Quantidade & Percentual \\
\hline AM & 1 & 3,03 \\
\hline BA & 1 & 3,03 \\
\hline CE & 2 & 6,06 \\
\hline DF & 1 & 3,03 \\
\hline ES & 1 & 3,03 \\
\hline MG & 3 & 9,09 \\
\hline PE & 1 & 3,03 \\
\hline PR & 3 & 9,09 \\
\hline RJ & 2 & 6,06 \\
\hline RN & 1 & 3,03 \\
\hline RS & 2 & 6,06 \\
\hline SC & 4 & 12,12 \\
\hline SP & 11 & 33,33 \\
\hline
\end{tabular}

